\title{
Acquisition of Human EEG Data during Linear Self-Motion on a Stewart Platform
}

\author{
Hugh Nolan, Robert Whelan, Richard B. Reilly \\ Trinity Centre for Bioengineering \\ Trinity Institute for Neuroscience \\ Trinity College Dublin, Ireland \\ [nolanhu, robert.whelan, richard.reilly]@tcd.ie
}

\begin{abstract}
The present study investigated the feasibility of acquiring electroencephalography (EEG) data during self-motion in human subjects. Subjects performed a visual oddball task - designed to evoke a P3 event-related potential - while being passively moved in the fore-aft direction on a Stewart platform. The results of this study indicate that reliable EEG data can be obtained during self-motion on a Stewart platform: this finding is important for the ecological validity of further research into human motion.
\end{abstract}

Keywords- electroencephalography; Self-motion; Stewart platform; vestibular; multisensory; P3

\section{INTRODUCTION}

\section{A. Background}

Multisensory integration (MSI) is an essential aspect of human function that is, as yet, poorly understood. Despite the fundamental role that sensory integration plays in performance and perception, how and when information from separate sensory modalities comes together in the human neocortex is an unsolved problem. The human sense of movement and balance integrates vestibular information with visual [ 1 ] and somatosensory information [2].

Understanding visual-vestibular sensory integration is key in understanding human balance. This is important because processing of vestibular signals deteriorates with age, which can cause postural instability and falls. Epidemiological studies show that falls are a very common problem in the older population with $30 \%$ of community dwelling elderly people $>65$ years of age falling each year and $12 \%$ of these falling at least twice [3][4][5]. Falls are the leading cause of injury-related hospitalization in over 65 's and hip fractures are the leading cause of mortality due to injury in people aged over 75 years [6]. Therefore, there is a need for a simple non-invasive method of measuring the vestibular system in vivo.

\section{B. Imaging methodologies for MSI}

There are many complexities involved in acquiring neural data from subjects who are moving. For example, neither magnetic resonance imaging (MRI) nor positron emitted tomography (PET) are

\author{
Heinrich H. Bülthoff, John S. Butler \\ Max Planck Institute for Biological Cybernetics \\ Tübingen, Germany \\ [heinrich.buelthoff, \\ john.butler]@tuebingen.mpg.de
}

suitable methods as the subject must remain in a fixed position during the procedure. Therefore, research on the neural correlates of linear selfmotion in humans has typically used visual vection, which is self-motion induced by large-field visual motion stimulation during which the stationary subject perceives the moving visual surroundings as being stable and themselves as moving [7][8]. Electroencephalography (EEG) would appear to be a suitable candidate for recording neural activity during motion because modern EEG acquisition equipment is lightweight and portable. Furthermore, a Stewart motion platform [9 ] provides an appropriate method of producing linear self-motion in a laboratory environment. Up until now, study of true self-motion in humans has been limited to rotational motion [10][11].

There are a number of potential drawbacks to using a Stewart motion platform in conjunction with EEG recording equipment. For example, noise could be introduced into the EEG signal from the motion of the actuators, the electrical noise of the platform power source, or from muscular activity of the subject as they compensate for the acceleration at the start and finish of the motion.

\section{Experimental paradigm}

In an attempt to address the potential issues described above, we first investigated the feasibility of recording EEG during motion by running a standard EEG task - an oddball paradigm - with four different motion conditions to address each of these potential issues.

An oddball paradigm is one in which occasional target stimuli have to be detected in a train of frequent irrelevant standard stimuli. It is known to evoke robust cognitive event-related potentials (ERPs; time-locked EEGs), which provide an objective and non-invasive tool for measuring the time-course of neural processing. Oddball paradigms evoke a $\mathrm{P} 3$ response [12] which is a positive ERP component, with a parietocentral scalp distribution, which typically peaks from 250 to $600 \mathrm{~ms}$ following an infrequent stimulus. The P3 has been investigated in a wide range of neurologic disorders, primarily because it can be reliably elicited with relatively simple paradigms. 
The four different levels of motion were termed static, idle, slow and fast. The static condition served as a control as there was no interference from the experimental setup and participant motion. In the idle condition the motors raised the platform to its neutral position - this is a centralized position that allows a full range of movement in all directions. The motors require power to maintain this position; thus allows investigation into the influence of electrical noise of the motors on the EEG signal. In the slow and fast conditions participants were sinusoidally moved forward and backwards at a frequency of $0.5 \mathrm{~Hz}$. These conditions are of most interest as there may be interference from the setup and use of the electrical motors, but also from the motion of the participant.

\section{METHODS}

\section{A. Participants}

Six male participants with normal or corrected-tonormal vision completed the experiment for payment. All participants except for the authors, $R W, J B$ and $H N$, were naïve to the purpose of the experiment. The average age was 25 years (range 18-31). Participants gave their informed consent before taking part in the experiment, which was performed in accordance with the ethical standards laid down in the 1964 Declaration of Helsinki.

\section{B. Apparatus}

The experiment was conducted in the Motion Lab at the Max Planck Institute for Biological Cybernetics, Tübingen, Germany. The main piece of equipment used was the Maxcue 600 platform manufactured by Motion-Base PLC [13]. This is a 6-legged platform controlled by hydraulics. It has 6 degrees of freedom, which are rotation and translation about 3 axes. The visuals were displayed on a projection screen, with a field of view of $86^{\circ} \times 65^{\circ}$ and a resolution of $1400 \times 1050$ with a refresh rate of 60 frames per second. Participants responded using a four-button response box.

EEG data were recorded using the BrainAmp MRplus $^{\mathrm{TM}}$ [14] electrode system from 8 scalp electrodes along the scalp midline and one EOG channel, digitized at $5000 \mathrm{~Hz}$. The system has a hardware highpass filter at $0.16 \mathrm{~Hz}$ and a hardware lowpass filter at $1000 \mathrm{~Hz}$ which were applied during data acquisition. EEGLAB was used for all EEG analyses [15]. The EEG data from the $\mathrm{Pz}$ channel were downsampled to $512 \mathrm{~Hz}$, bandpass filtered between $2-45 \mathrm{~Hz}$, epoched from -1000 to $2000 \mathrm{~ms}$ after stimulus presentation, and baseline corrected from $200 \mathrm{~ms}$ before stimulus presentation. Data were manually scanned for obvious artifacts (e.g., muscle twitch).
Furthermore, these experiments were later replicated using a Biosemi ActiveTwo $^{\mathrm{TM}}$ [16] 128 channel EEG system for one subject. This was important because the Biosemi, unlike the MRplus, is an unshielded system. It was possible, therefore, that electrical noise from the Stewart platform would interfere with the data from the unshielded system. The data acquired with the Biosemi were digitized at $2048 \mathrm{~Hz}$, with a hardware lowpass filter at $400 \mathrm{~Hz}$ applied during data acquisition. They were downsampled to $256 \mathrm{~Hz}$. The data from the $\mathrm{Pz}$ channel was epoched from $1000 \mathrm{~ms}$ pre-stimulus to $2000 \mathrm{~ms}$ post-stimulus, baseline-corrected from $-500 \mathrm{~ms}$ to stimulus onset, and then filtered between 2 and $45 \mathrm{~Hz}$. For the BrainAmp and Biosemi data, poor quality epochs were identified and rejected, using an automated method based on epoch amplitude, deviation and variance.

Following this step, specifically for the Biosemi data, Independent Component Analysis (ICA) was performed, and noisy and artifactual components were identified and rejected by an automated method based on a component's maximum component standard deviation, correlation with the EOG channels, contribution to the EEG waveform, spectral power and spectral slope. ICA was not feasible for the BrainAmp data due to the low number of channels, which would not allow for a good approximation of statistical independence.

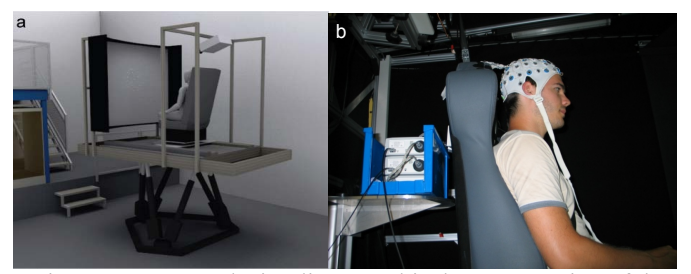

Figure 1. Setup and stimuli. a Graphical representation of the Stewart motion platform. b Experimental setup.

\section{Visual oddball paradigm}

The visual oddball paradigm consisted of different sized circles presented for $60 \mathrm{~ms}$ on the screen, separated by an inter-stimulus interval of $2000 \mathrm{~ms}$, for 205 trials in a pseudorandom order. Frequent non-target $(80 \%)$ and infrequent target $(20 \%)$ circles differed in size and subtended 2 degrees and 6 degrees, respectively. Subjects were instructed to press a button as quickly as possible following a target (large) circle. The visuals were displayed using Presentation ${ }^{\mathrm{TM}}$.

\section{Motion Stimuli}

There were four different motion stimuli: static, idle, slow and fast. In the static condition the platform was turned off. In the idle condition the platform was raised to its neutral position then remained idle in this position. In the slow and fast conditions the platform was moved sinusoidally in a fore-aft motion at a frequency of $0.5 \mathrm{~Hz}$ for 230 
cycles at different maximum accelerations of $0.1 \mathrm{mG}$ and $0.5 \mathrm{mG}$, respectively. The motions were controlled by Virtools ${ }^{\mathrm{TM}}$.

\section{E. Experiment procedure}

Participants performed the visual oddball task during all four motion conditions (static, idle, slow and fast). In the idle, slow and fast conditions the motion platform was raised to the neutral position and motion was started before the beginning of the oddball paradigm. The timing of the onset of the visual stimulus was pseudorandom to ensure it was not locked to motion stimulus.

\section{RESULTS}
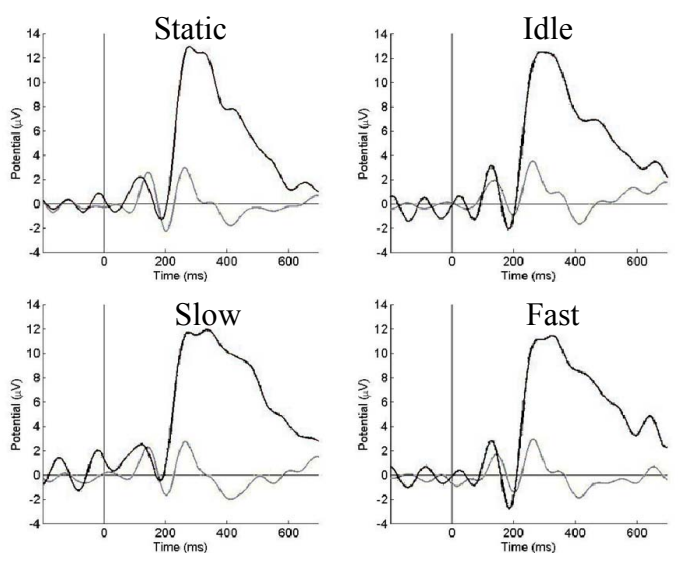

Figure 2. Averaged ERP data $(\mathrm{n}=5)$ recorded under all motion conditions from $\mathrm{Pz}$ acquired using BrainAmp MRplus. Both target (black) and standard (grey) averages are shown.

TABLE 1

P300 CORRELATION COEFFICIENTS BETWEEN MOTION CONDITIONS

\begin{tabular}{llll}
\hline & Idle & Slow & Fast \\
Static & 0.9766 & 0.9394 & 0.9682 \\
Idle & & 0.9326 & 0.9687 \\
Slow & & & 0.9488 \\
\hline
\end{tabular}

Figure 2 shows the average ERP data $(n=5)$ for each motion condition. A robust and characteristic peak around $300 \mathrm{~ms}$ can be seen in all the target (grey) ERPs. Table 1 shows the correlation coefficients during the interval $-100 \mathrm{~ms}$ to $600 \mathrm{~ms}$ of the ERPs, which can all be seen to be above 0.93 . For display purposes, the ERP data in all figures have been subject to a $13 \mathrm{~Hz}$ low-pass filter.

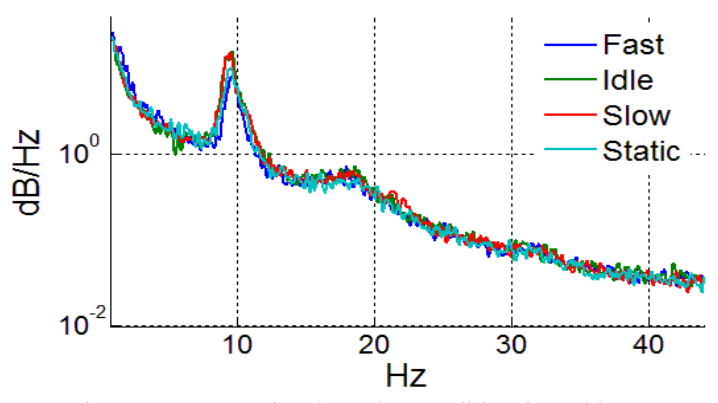

Figure 3. Spectra of each motion condition for Subject 2.

Figure 3 shows the spectra for each of the motion conditions for a representative subject (Subject 2). The spectra were subject to a 20-point moving average smoothing filter for display purposes, as there were a large number of data points in the resultant FFT. These spectra produce a covariance matrix with a mean of $5.9823 \pm 0.5226$, and a variance of 0.2731 .

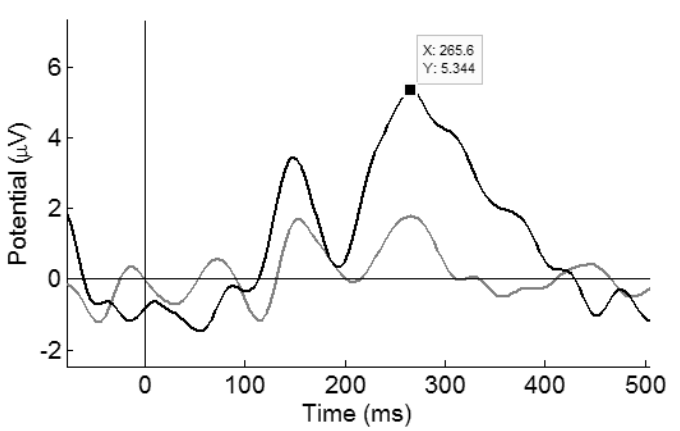

Figure 4. ERP data $(n=1)$ recorded using the Biosemi ActiveTwo system during the Idle condition. The target (black) and standard (grey) are shown.

Figure 4 shows a P3 recorded with the Biosemi system.

On inspecting epochs, we found there was no rejection required for the BrainAmp data. The automated rejection tool selected 5 out of 5536 epochs across all 5 subjects, from a static, slow and fast dataset for the Biosemi data.

\section{DISCUSSION}

We found that there was minimal interference to the EEG signal during motion of the participants on the platform, using both the shielded MRplus system and the unshielded Biosemi system. A reliable P3 ERP was evoked in all conditions, as demonstrated by the peak around $300 \mathrm{~ms}$ in the target ERP (black), which was not present in the standard ERP (grey). Furthermore, as Table 1 shows, the correlation between the static (motorsoff) condition and the other 3 conditions is similar to the correlation between the motors-on conditions, indicating that the recorded data are unaffected by the platform's state. This is also reflected on examination of the rejected epochs, which show no electromagnetic artifacts caused by 
the Stewart platform, and negligible difference in rejection rates across different motion conditions.

More evidence can be seen by inspecting Figure 3, which shows the spectra of each of Subject 2's sessions, overlaid. There are no large deviations from the overall trend, as can be seen by visual inspection, and shown in the small standard deviation and variance in the covariances between the spectra. These properties are seen in all subjects.

Figure 4 shows a sample plot from the Biosemi P3 recordings. The peak at $300 \mathrm{~ms}$ is present but not as well-defined as those in Fig. 2; as Fig. 4 depicts only a single subject, more testing will be required to determine whether this is due to inter-subject variability or a lower quality signal in the Biosemi.

These results are of interest because we have shown that EEG can be acquired during linear selfmotion, which has never been demonstrated before in humans. The methodology described in this study may provide a basis for investigating the functioning of the vestibular system. In particular, the combined use of a Stewart platform and EEG recording could facilitate the investigation of agerelated disorders of the vestibular system, and vestibular functional decline. This method may also be useful for further investigating MSI in the vestibular system. Future experiments will investigate the relationship between the visual and vestibular system via a combined Visual-Vestibular P3 experiment.

\section{REFERENCES}

[1] H. Straka, N. Dieringer, Basic organization principles of the VOR: lessons from frogs, Progress in Neurobiology, Vol. 73(4), pp. 259-309, July 2004.

[2] F. B. Horak, G. M. Earhart V. Dietz, Postural responses to combinations of head and body displacements: vestibular-somatosensory interactions, Exp Brain Res, Vol. 141, pp. 410-414, 2001.

[3] J. O'Loughlin et al. Incidence of and risk factors for falls and injurious falls among the communitydwelling elderly, American journal of epidemiology, Vol. 137, pp. 342-54, 1993.

[4] M. E. Tinetti, M. Speechley, Prevention of falls among the elderly, New England journal of medicine, Vol. 320, pp. 1055-1059, 1989.

[5] S. R. Lord, D. McLean, G.

Strathers, Physiological factors associated with injurious falls in older people living in the community, Gerontology, Vol. 38, pp. 338-346, 1992.

[6] World Health Organization, Assessment of fracture risk and its application to screening for postmenopausal osteoporosis, Technical report series 843, Geneva, 1994.
[7] G. Kovacs, M. Raabe, and M. W. Greenlee, The fMRI correlates of visually induced self-motion in depth, Cereberal Cortex, Vol. 18, pp. 1779-1787, 2008.

[8] T. Brandt, P. Bartenstein, A. Janek, and M Dieterich. Reciprocal inhibitory visualvestibular interaction, Reciprocal inhibitory visualvestibular interaction. Visual motion stimulation deactivates the parieto-insular vestibular cortex, BRAIN, Vol. 121, pp. 1749-1758, 1998.

[9] D. Stewart, A Platform with Six Degrees of Freedom, UK Institution of Mechanical Engineers Proceedings, Vol. 180, Pt 1, No 15, 1965-66.

[10] D. Schneider, C. Kolchev, L. Constantinescu, C.-F. Claussen, Vestibular Evoked Potentials and Brain Electrical Activity Mapping - A Test of Vestibular Function - A Review, Int. Tinnitus Journal, Vol. 2, pp. 27-43, 1996.

[11] J. D. Hood, A. Kayan, Observations upon the evoked responses to natural vestibular stimulation, Electroencephalography and Clinical

Neurophysiology, Vol. 62, pp. 266-276, 1985. [12] S. Sutton, M. Braren, J. Zubin, E. R John, Evoked-Potential Correlates of Stimulus Uncertainty, Science, 150(3700), pp. 1187-1188, 1965.

[13] M. von der Heyde, A Distributed Virtual Reality System for Spatial Updating: Concepts, Implementation, and Experiments, $\mathrm{PhD}$ thesis, Universität Bielefeld - Technische Fakultät, 2001. [14] http://www.brainproducts.com/ - Brain Products $\mathrm{GmbH}$.

[15] A. Delorme, S. Makeig, EEGLAB: an open source toolbox for analysis of single-trial EEG dynamics including independent component analysis, J. Neurosci Methods, Vol. 134, pp. 9-21, 2004.

[16] http://www.biosemi.com/ - Biosemi company 